\title{
Estado Actual de la Cacaocultura: Una Revisión de sus Principales Limitantes.
}

\section{Resumen}

El cultivo del cacao (Theobroma cacao L) ha adquirido gran importancia para Colombia en los últimos años, debido al incremento de las áreas cultivadas y al compromiso con su exportación por parte del Gobierno nacional. Para el año 2018, en el país había aproximadamente 170.000 hectáreas sembradas. Esta especie se cultiva entre los $20^{\circ}$ de latitud norte y los $20^{\circ}$ de latitud sur, a altitudes comprendidas entre los 0-1200 msnm. Los principales productores de cacao se encuentran ubicados en el continente africano (Ghana y Costa de Marfil), que contribuyen aproximadamente con el $70 \%$ de la producción mundial. En América, la producción se enfoca en cultivos de cacao fino y de aroma, especialmente en países como Ecuador, Perú, Brasil, Colombia, México, entre otros. Los retos que enfrenta este cultivo en la actualidad son diversos: la escasa disponibilidad de mano de obra, la existencia de enfermedades limitantes como las causadas por Moniliophthora roreri y Phythophthora spp., y las restricciones establecidas por los países consumidores frente a la presencia de ciertos metales pesados que son bioacumulados por el cacao y que pueden generar problemas a la salud humana. Por tanto, el objetivo de esta revisión es analizar las diferentes variables que intervienen en este cultivo, como instrumento de consulta para productores y técnicos interesados en este sistema productivo, con el fin de que se convierta en una herramienta en la toma de decisiones del cultivo y de sus implicaciones de establecimiento.

Palabras clave: economía rural, enfermedades, metales pesados, plagas, sistema productivo, Theobroma cacao.

\section{Current State of Cocoa Plantation:}

\section{A Review of its Main Limitations}

\section{Abstract}

The cocoa (Theobroma cacao L) crop has become very important for Colombia in recent years, due to the increasing in cultivated areas and the exportation commitment of the national government. For 2018, there were in the country approximately 170,000 planted hectares. This species is cultivated between $20^{\circ}$ north latitude and $20^{\circ}$ south latitude, at altitudes between 0-1200 m.a.s.l. The main cocoa producers are located in the African continent (Ghana and Ivory Coast), which contribute approximately with $70 \%$ of world production. In America, the production focuses on fine and aroma cocoa crops, especially in countries such as Ecuador, Peru, Brazil, Colombia, Mexico, among others. Currently, this crop faces diverse challenges: scarce availability of labor, the existence of limiting diseases such as those caused by Moniliophthora roreri and Phythophthora spp., and the restrictions established by consuming countries due to the presence of certain heavy metals that are bioaccumulated by cocoa, and that can cause problems for human health. Therefore, the objective of this review is to analyze different variables that intervene in this crop as a consultation tool for producers and technicians interested in the cocoa production system, so that it becomes a decision-making instrument for the crop cultivation and the starting up implications.

Keywords: rural economy, diseases, heavy metals, pests, production system, Theobroma cacao.

1 Corporación colombiana de Investigación Agropecuaria - Agrosavia (Santander, Colombia), eantolinez@agrosavia.co; eduarsandoval1@yahoo.com. (1) ORCID: 0000-0003-0145-6002

2 Universidad Pedagógica y Tecnológica de Colombia (Tunja Colombia), pedro.almanza@uptc.edu.co, (D) 0RCID: 0000-0002-9207-0617

3 Corporación colombiana de Investigación Agropecuaria - Agrosavia (Santander- Colombia), abarona@agrosavia.co, (D) 0RCID: 0000-0001-8487-2727

4 Corporación colombiana de Investigación Agropecuaria - Agrosavia (Tolima, Colombia), epolanco@agrosavia.co, (D) 0RCID: 0000-0001-9229-4074

5 Universidad Pedagógica y Tecnológica de Colombia (Tunja Colombia), pablo.serrano@uptc.edu.co, (DD ORCID: 0000-0002-1270-3024 


\section{I.Introducción}

El cacao (Theobroma cacao $L$ ) es un cultivo importante en el desarrollo de la economía de algunos países del área ecuatorial (Acosta et al., 2018). Su producción en la cosecha 2016-2017 fue de 4,7 millones de toneladas en el mundo (Lu et al., 2018). Sin embargo, para el año 2016, estas zonas eran responsables de solamente el $14 \%$ de la producción mundial (Campos, Nieto \& Oomah, 2018). En la actualidad, los tres principales productores son Costa de Marfil (1.963.949 t), Ghana (947.632 t) e Indonesia (593.832 t), que aportaron el 66,73 \% de la producción mundial en el año 2018 (Faostat, 2018; Lu et al., 2018; Campos et al., 2018).

El cacao pertenece a la familia Sterculiaceae y al género Theobroma; este género tiene 22 especies, de las cuales T. cacao L. es comercialmente la más importante debido al valor económico de sus semillas (Kongor et al., 2016). Los mismos autores mencionan que el cacao es cultivado en más de $70.000 \mathrm{~km}^{2} \mathrm{en}$ el mundo entre los $20^{\circ}$ de latitud norte y sur; es una especie perenne, que en su mayoría de variedades requiere de sombra (Hulme et al., 2018). Una de sus principales características es la de ser cauliflora, con polinización cruzada y monoica. En estado silvestre, los árboles de cacao pueden alcanzar hasta $25 \mathrm{~m}$ de altura (Lachenaud, Paulin, Ducamp \& Thevenin, 2007).

Algunas de las nuevas variedades de cacao están adaptadas para tener altas producciones en sistemas de libre exposición, pero este tipo de cultivos requiere de un mayor uso de insumos pesticidas y fertilizantes (Niether, Schneidewind, Fuch, Schneider \& Armengot, 2019; Espinosa-Alzate \& Ríos-Osorio, 2016) y mayor suministro hídrico (De Araújo et al., 2017).

Shavez, Ahmad, Jan y Bashir (2017) mencionan que los principales tipos de cacao (Figura 1) son: el criollo, considerado el de mejor calidad por su sabor medio, notas muy aromáticas y florales y por presentar almendra blanca; el forastero, que se caracteriza por ser una variedad robusta y resistente a condiciones adversas, es la materia prima de aproximadamente el 80 $\%$ de la industria chocolatera al proveer los precursores de notas "básicas"; y el trinitario, que es una combinación del criollo y el forastero, caracterizado por presentar notas de vino (Quiroz \& Fogliano, 2018).

El principal atributo considerado para la comercialización del cacao es el aroma. El criollo y el trinitario se definen como cacaos finos y de aroma (con calidad y precio de comercialización mayor), mientras que el cacao forastero se considera como cacao tipo bulk/ordinario (Castro, Idrogo-Vázquez, Siche \& Cárdenas 2019).
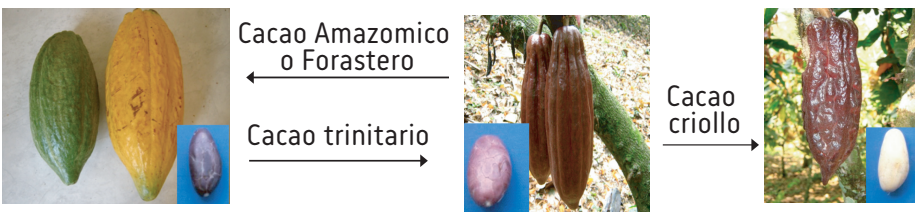

Figura 1. Tipos de cacao (criollo, trinitario y forastero).

Se pueden obtener muchos productos del cacao, después de la fermentación y el procesamiento de los granos; como pasta fluida o licor (de ahí se hace el chocolate), manteca de cacao y el cacao en polvo. El chocolate se puede usar como barra para consumo directo o como chocolate para hornear; el cacao en polvo es usado por confiteros y panaderos para el recubrimiento de barras de caramelo, galletas y pasteles; además, el cacao en polvo es materia prima para la industria de los helados (Beg, Ahmad, Jan \& Bashir, 2017).

Las exportaciones de los derivados del cacao incluyen el cacao en grano, que representa un $27 \%$, la manteca, el $8 \%$, la pasta fluida o el licor, el $7 \%$, el polvo, el $9 \%$ y el chocolate, el $49 \%$. Para el año 2014 el mayor exportador de chocolate procesado fue el continente europeo representando el $69 \%$ (Arvelo et al., 2016).

Los principales productores de cacao en el mundo comparten dificultades similares que les impiden aumentar la productividad. Ellos indican que estas tienen que ver principalmente con bajos precios de comercialización en finca, altos precios en los insumos requeridos, falta de acceso a los créditos, producción en minifundio, aspectos que desencadenan inadecuados manejos de plagas y enfermedades, deficientes labores de mantenimiento y bajas aplicaciones de nutrientes, y traen como resultado bajas producciones por el agotamiento de los suelos, debido a la pérdida de la fertilidad natural de estos (McMahon et al., 2015; Wessel \& Quist, 2015).

Dentro de las actuales dificultades del cultivo de cacao se encuentra la presencia de metales pesados (principalmente cadmio), tanto en almendra como en producto transformado (Gramlich et al., 2018). Esto debido a que la planta bioacumula el metal en la almendra. Según la reglamentación de la Unión Europea, las máximas concentraciones permitidas en suelos agrícolas son de $3 \mathrm{ppm}$ para cadmio y $300 \mathrm{ppm}$ para plomo (Huamaní, Huauya, Mansilla, Florida \& Neira, 2012). En el caso de la almendra, el límite permitido por la Cooperación Técnica Alemana (GTZ, por sus siglas en alemán) es de 0,05 mg kg-1 (Acosta \& Pozo, 2013). En el caso de Colombia, el Instituto Nacional de Vigilancia de Medicamentos y Alimentos (INVIMA) en el 2016-2017 generó un programa nacional de vigilancia y control de cadmio en productos derivados del cacao y planteó 
que el licor de cacao debería tener un contenido de cadmio máximo de $5 \mathrm{mg} \mathrm{kg-1}^{-1}$ (INVIMA, 2016)

Teniendo en cuenta que no existen productos que sustituyan las cualidades organolépticas, o cultivos de los cuales se pueda hacer chocolate, existe la perspectiva que la demanda de este tipo de materia prima crezca (Utomo, Prawoto, Bonnet, Bangviwat \& Gheewala, 2016), pues actualmente el chocolate se considera como un producto de lujo (Castro et al., 2019). En los últimos cincuenta años la demanda mundial de cacao ha aumentado a una tasa de $2,5 \%$ anual, debido al crecimiento del consumo en mercados emergentes como China e India (Singh, Sanderson, Field, Fidelis \& Yinil, 2019).

La presente revisión tiene como objetivo analizar las diferentes variables que intervienen en este cultivo, para que sea usado como herramienta de consulta y toma de decisiones previas a la puesta en marcha por productores y técnicos interesados en este sistema productivo.

\section{II.Generalidades del cultivo.}

El árbol de cacao (Theobroma cacao L.) es nativo del sotobosque de la selva amazónica, por esta característica su desarrollo se da bien bajo un dosel de arboles de sombra y también bajo diversos sistemas agroforestales (Niether et al., 2019). Una de las principales limitantes para su cultivo es la disponibilidad hídrica, pues el patrón de cultivo está relacionado con altas precipitaciones, debido a que la planta de cacao es susceptible a la sequía (Adams, Acheampong, Kyereh \& Kyereh, 2017).

Shavez et al. (2017) afirman que la producción de cacao varía de acuerdo con el país y el tipo de cacao que se cultive (Tabla I); la producción abarca desde sistemas de monocultivo a plena exposición hasta sistemas agroforestales muy diversos (Niether et al., 2017).

Colombia, para el periodo de cosecha 2018, contaba con un área sembrada de cerca de 176.050 ha y con producción de $56.867 \mathrm{t}$ año- ${ }^{-1}$. Siendo el departamento de Santander el mayor productor, con el $41,4 \%$ de la producción nacional, seguido por Antioquia con $8,6 \%$ y Arauca con $7,9 \%$ (Fedecacao, 2019). Las siembras en estas zonas se han realizado con materiales híbridos trinitarios y amazónicos, descendientes de híbridos y clones introducidos (Agudelo-Castañeda, Cadena-Torres, Almanza-Mechán \& Pinzón-Sandoval, 2018).

\section{Tabla 1. Producción global de cacao}

\begin{tabular}{|c|c|c|c|}
\hline Zona del planeta & Países & $\begin{array}{l}\text { Estimado de } \\
\text { producción (kg } \\
\left.\mathrm{ha}^{-1}\right)\end{array}$ & $\begin{array}{l}\text { Participación en } \\
\text { la producción (\%) }\end{array}$ \\
\hline África & $\begin{array}{l}\text { Costa de Marfil, Ghana, } \\
\text { Nigeria, Camerún }\end{array}$ & $300-400$ & 68 \\
\hline Asia/Oceanía & $\begin{array}{l}\text { Papúa Nueva Guinea, } \\
\text { Malasia, Indonesia }\end{array}$ & $\begin{array}{l}\text { Alrededor de } \\
500\end{array}$ & 17 \\
\hline América & Colombia, Brasil, Ecuador & $500-600$ & 15 \\
\hline
\end{tabular}

En este sentido, existe una diversa variabilidad genética adaptada a los diferentes parámetros climáticos que se presentan en las zonas agroecológicas del país, lo que permite ser cultivado bajo condiciones de diferentes sistemas de producción, en asocio con especies como fríjol (Phaseolus vulgaris L.), yuca (Manihot esculenta Crantz.), plátano (Musa sp.), como sombríos transitorios; o con sistemas de sombríos permanentes con árboles frutales o maderables, los cuales le permiten al agricultor tener fuentes de ingreso adicionales (Mejía \& Palencia, 2004).

La implementación de sistemas productivos a plena exposición podría acarrear inconvenientes ecológicos, como alteración de la calidad del suelo, pérdida de la biodiversidad, degradación de los bosques, emisión de gases de efecto invernadero que afectan directamente la calidad del suelo y el rendimiento del cultivo (Ebagnerin et al., 2015).

\section{III.Principales plagas y enfermedades del cacao.}

Las enfermedades de los cultivos son un problema creciente en el mundo, se estima que por su efecto se pierde entre un 20-40 \% de la productividad global (Andres et al., 2018); aunque se espera que la demanda de cacao siga creciendo (Utomo et al., 2016), la productividad de este ha disminuido considerablemente a causa de la reducción de la fertilidad natural del suelo, aumento de plagas y enfermedades y un clima cada vez más seco y cálido (Blaser, Oppong, Yeboah \& Six, 2017). Las principales enfermedades y plagas que afectan el cultivo son: Moniliasis, Phytopththora, virus de los brotes inflamados del cacao (CSSVD), y perforadores del fruto (Jaimes \& Aranzazu 2010; Andres et al., 2017).

\section{A.Moniliasis}

La pudrición de la vaina helada, moniliasis, monilia, mancha helada, nieve, o pasmo, como es conocida la enfermedad, es causada por el hongo Moniliophthora roreri (Cif. \& Par), considerada la principal enfermedad que afecta los cultivos de cacao en Colombia y otros países de América central y Suramérica (Cubillos, 2017). Díaz-Valderrama y Aime (2016) mencionan que han sido descritas dos especies de M. roreri, que se diferencian principalmente por su tamaño de espora y el huésped, M. roreri var. Roreri, que produce pequeñas esporas e infecta principalmente a especies de los géneros Theobroma y Herrania, mientras que M. roreri var. Gileri produce esporas grandes y tiene a T. gileri como única especie huésped.

Los daños por esta enfermedad en Colombia alcanzan más del $40 \%$ de la producción anual del país (Cárdenas, Darghan, Sosa \& Rodríguez, 2017), en términos de pérdidas de grano comer- 
cial significaría 28.000 t sobre una producción aproximada de 42.000 t (Correa, Castro \& Coy, 2014). Su presencia en algunos países de América como Cuba se considera como de tipo cuarentenario (Martínez \& Pérez, 2015).

Correa et al. (2014), Evans (2016) y Jaimes y Aranzazu (2010) sugieren diversas medidas de control contra este patógeno. Estas incluyen un control cultural a través de la remoción de frutos infectados antes de la esporulación del patógeno (cada siete días), podas de mantenimiento por lo menos dos veces al año. Algunos productos de síntesis química de tipo protectante (a base de cobre) pueden ser combinados con la medida cultural en las épocas de alta incidencia de la enfermedad, aplicándose semanalmente durante dos meses, iniciando en la época de mayor floración (Jaimes \& Aranzazu, 2010). En el aspecto biológico, los anteriores autores manifiestan que se han llevado a cabo diferentes estudios con micoparásitos; sin embargo, estas medidas han funcionado para disminuir la incidencia de la enfermedad, pero no su erradicación. Un último aspecto para tener en cuenta es el control genético, dado que se convierte en la mejor opción para adoptar por parte del productor; sin embargo, son escasas las investigaciones en materiales genéticos que presenten resistencia a la enfermedad (Jaimes \& Aranzazu, 2010).

Teniendo en cuenta lo anterior, para el control se requieren conocimientos sobre la fase biotrófica del patógeno, que serían importantes para entender completamente su dinámica y los factores que inician la conversión de biotrófo a necrotófo (Bailey, Ali, Strem \& Meinhardt, 2018; Jaimes et al., 2016).

\section{B.Phytopththora}

Conocida como la vaina negra de cacao, causada por algunas especies del género Phytophthora (P. palmivora, P. megakarya, P. capsici, P. citrophthora, P. nicotianaevar. Parasitica, P. megasperma y P. arecae) es también una de las enfermedades más limitantes en el cultivo (Akrofi, Amoako, Assuah \& Asare, 2015; Legavre et al., 2015; Instituto Colombiano Agropecuario [ICA], 2012); McMahon et al. (2015) señalan que las pérdidas por esta enfermedad pueden llegar hasta el $50 \%$ de la producción e irse incrementando con periodos particularmente húmedos; si la incidencia de esta enfermedad coincide con la temporada principal de cosecha, se puede convertir en un problema grave para los productores.

El patógeno puede infectar diferentes estados de desarrollo de la planta, causando tizón en plántulas y tejidos vegetativos de árboles maduros, cancro en el tallo, y en las vainas de los árboles ocasionar la pudrición de la vaina de Phytophthora (Ppr, o vaina negra) (Purwantara et al., 2015).

Ramírez (2016) hizo seguimiento a la evolución de la enfermedad durante varios años en una finca de Colombia y encontró que la incidencia y la severidad variaban de acuerdo con condi- ciones como edad del árbol (mayor incidencia en árboles de mayor edad), ubicación del lote (alta heterogeneidad) explicada en diferentes altitudes evaluadas (1000-1300 msnm), topografía de montaña (donde se pueden presentar diferentes microclimas).

Las pérdidas reportadas por Ramírez (2016) equivalen en promedio durante los tres años de evaluación a $81,27 \mathrm{~kg}$ ha-1 año-1. Si se analiza donde una unidad agrícola familiar necesita mínimo cuatro hectáreas para obtener rentabilidad en el cultivo y teniendo como base los precios reportados por Agronet (2020), para marzo se tendría una pérdida aproximada de 2.714.418 pesos colombianos. Teniendo en cuenta que las labores realizadas en la investigación de Ramírez (2016) estaban enfocadas en un manejo agronómico, se esperaría que estas pérdidas aumenten en lotes donde el manejo no es el adecuado.

Numerosos estudios se han llevado a cabo en la búsqueda de un control efectivo de este patógeno. Por ejemplo, Ndoungue et al. (2018) reportan medidas como la búsqueda de variedades mejoradas, control cultural, aplicaciones de agroquímicos (especialmente a base de $\mathrm{Cu}$ ), aplicaciones de algunos antagonistas como los del género Trichoderma, pero hasta el momento ninguna de las investigaciones reporta eliminación total del patógeno.

C.Virus de los brotes inflamados del cacao (CSSVD)

La CSSVD (Cocoa Swollen Shoot Virus Disease, por sus siglas del inglés) es uno de los factores que más ha limitado la productividad de los cultivos de cacao en el oeste africano por más de ochenta años, siendo particularmente severa en Ghana donde se ha observado desde 1922 (Andres et al., 2017).

La identificación de plantas infectadas por el virus se ha basado principalmente en el análisis de los síntomas visuales. Sin embargo, las deficiencias nutricionales también pueden presentar síntomas similares a las de la infección producida por el virus, por lo que el diagnóstico podría ser engañoso (Obok, Wetten \& Allainguillaume, 2018). Los mismos autores señalan que una alternativa para el diagnóstico temprano de la enfermedad es el estudio molecular de algunas cochinillas que son consideradas como los principales vectores de la enfermedad, los avances en este campo permitirán un diagnóstico precoz antes que la planta de cacao empiece a manifestar los síntomas visibles.

\section{D.Perforadores del fruto de cacao}

En los últimos años en el continente americano se han disparado las alertas a causa de insectos que perforan el fruto del cacao, causando una serie de daños, tanto a la almendra como a los procesos poscosecha (fermentación previa de las almendras). Son diversas las especies de insectos que atacan el fruto del cacao, en su mayoría las identificadas son del orden Lepidoptera (ICA, 2017; Instituto Nacional de Investigaciones 
Agrícolas [INIA], 2004).

Carabalí, Senejoa y Montes (2018) citan dos especies de importancia económica en cuanto al daño producido a frutos de cacao: Carmenta foraseminis y Carmenta theobromae, de las cuales la primera es considerada la más limitante en cultivos en Panamá, Venezuela, Brasil y Colombia, dado que se caracteriza por barrenar el material placentario y las semillas del fruto. Dentro de los posibles controles para este tipo de insectos se encuentran los reportados por Figueroa, Ramírez, Katil y Rieche(2013), donde los aislamientos nativos de Paecilomyces sp. y Lecanicillium sp. demostraron resultados patogénicos para larvas del insecto $C$. foraseminis en último estadio.

Por su parte, Carabalí et al. (2018) presentan un análisis detallado de manejo agronómico que va desde el monitoreo hasta el manejo de trampas, atrayentes, medidas de tipo cultural (remoción y solarización de frutos afectados) y utilización de algunos enemigos naturales como especies de Trichograma sp., Telenomus sp., Brachymeria sp., Polistes sp., y un hongo entomopatógeno (Paecelomyces sp).

\section{IV.Problemas de contaminación}

\section{por metales pesados.}

El cultivo de cacao absorbe metales pesados que se encuentran en el suelo y los acumula en las semillas. En el caso de metales como el cadmio y el plomo, estos se encuentran presentes en la corteza terrestre de manera natural en forma de minerales 0 han sido introducidos por acción antropogénica, siendo absorbidos por las plantas independientemente de su origen (Huamaní et al., 2012).

Las plantas cultivadas representan una de las principales formas de translocación de los metales pesados desde los suelos hasta los seres humanos, dado que estos pueden acumularse en los tejidos que son consumidos y afectar la calidad de la dieta (Rodríguez, Darghan \& Henao, 2019).

La contaminación por cadmio (Cd), plomo y otros metales pesados representa uno de los mayores riesgos para la salud humana y su seguridad alimentaria (Chávez et al., 2015; Rodríguez et al., 2019), debido a que sus altos niveles en el organismo humano pueden estar relacionados con la aparición de enfermedades crónicas como disfunción renal, formación de cálculos renales, defectos respiratorios, esqueléticos, reproductivos y endocrinos y alteraciones del metabolismo del calcio (Arévalo, Arévalo, Baligar \& He, 2017). Al respecto, Kruszewski, Obiedzinski y Kowalska (2018) afirman que la Agencia Internacional para la Investigación del Cáncer (IARC, por sus siglas en inglés) ha clasificado el cadmio como cancerígeno para humanos (grupo 1).

La acumulación de cadmio por parte de las plantas de cacao ha sido recientemente de interés público, después de que se encontraran productos a base de cacao con altos contenidos de este metal (> $100 \mu \mathrm{g} \mathrm{kg-1}{ }^{-1}$ de Cd) (Gramlich et al., 2018).

Son diversas las formas como el suelo cultivable se puede contaminar con cadmio, desde la misma meteorización de las rocas, la acumulación de ciertos lodos, el estiércol de ganado y la contaminación antropogénica proveniente de actividades industriales y realización de prácticas de aplicación de fertilizantes fosfatados (Lewis, Lennon, Eudoxie \& Umaharan, 2018). De acuerdo con las regulaciones de la Unión Europea, se han definido los rangos máximos de contenido permisible de plomo y cadmio en el chocolate y cacao en polvo comercializados en Europa (Tabla II).

Diferentes investigaciones han encontrado que durante el proceso de beneficio de la almendra de cacao pueden ocurrir variaciones fisicoquímicas del grano como consecuencia del tipo de fermentador utilizado y su tiempo de secado, que afectan las concentraciones de los metales y la calidad del producto final (Casteblanco, 2018).

Tabla 2. Contenidos máximos permisibles de cadmio en el chocolate (CODEX Alimentarius, 2015). Sobresale el alto valor asignado para chocolates con porcentaje total de materia seca $\geq 50 \%$

\begin{tabular}{lcc}
\hline Producto & Porcentaje total materia seca & $\begin{array}{c}\text { Límite máximo de cadmio } \\
\text { permitido** }\end{array}$ \\
\hline Chocolate de leche & $<30 \%$ & $0,10 \mathrm{mg} / \mathrm{kg}$ \\
Chocolate de leche & $\geq 60 \%$ & $0,30 \mathrm{mg} / \mathrm{kg}$ \\
Chocolate & $<50 \%$ & $0,30 \mathrm{mg} / \mathrm{kg}$ \\
Chocolate $_{\text {Cacao en polvo }}^{*}$ & $\geq 50 \%$ & $0,80 \mathrm{mg} / \mathrm{kg}$ \\
\hline
\end{tabular}

*Este límite aplica tanto al chocolate en polvo o cacao en polvo vendido al consumidor final, como al usado como ingrediente en cacao en polvo edulcorado vendido al consumidor (chocolate para tomar).

** Estos límites empezarán a regir a partir de 1 de enero de 2019.

Fuente: Casteblanco (2018)

Se detallan varios estudios donde se caracteriza la absorción de cadmio por las plantas de cacao, los niveles naturales en el suelo y los niveles encontrados en producto procesado (Romero-Estévez, Yánez-Jacome, Simbaña-Farinango \& Navarrete, 2019; Kruszewski et al., 2018; Lo Dico et al., 2018; Argüello et al., 2019).

Gramlich et al. (2017) observaron la captación de Cd por parte de las plantas de cacao en sistemas agroforestales y de monocultivo, aunque se presentaron diferencias estadísticas comparando sistema agroforestal cultivado con el material genético TSH 565, que se caracterizó por acumular menor cantidad de Cd en la hoja de los árboles, cotejado con el sistema a libre exposición cultivado con ICS 1. No se pudo concluir que alguno fuese mejor, pues no fue posible separar en el análisis estadístico la variable tipo de sombrío de la variable material genético. 


\section{V.Economía rural alrededor del cacao}

La dinámica de la pobreza rural ha sido objeto de diversos estudios y políticas; sin embargo, no se ha hecho un compromiso directo para establecer la relación entre la intensificación de la agricultura de manera sostenible y una comprensión más matizada de la pobreza como un fenómeno multidimensional y socialmente desagregado (Hirons et al., 2018).

Una de las dificultades que se presentan de manera general en los productores es que solo tienen una visión a corto plazo, no solo en el manejo del dinero, sino también en aspectos relacionados con el manejo del cultivo, lo cual se encuentra relacionado con una variación en el precio, pues está demostrado que a medida que aumenta el valor del producto, se incrementan los cuidados al sistema productivo (Montoya, Montoya \& Lowy, 2015).

Existen alrededor de 570 millones de espacios productivos familiares en todo el mundo, que producen el $80 \%$ del suministro mundial de alimentos; de estas fincas familiares, aproximadamente el $72 \%$ corresponde a predios con menos de una hectárea (Curry et al., 2015).

El cultivo de cacao en el siglo XXI se ha convertido en el foco de atención de medios de comunicación y agencias gubernamentales principalmente por la existencia de condiciones laborales irregulares. Los principales estudios que están relacionados con esta problemática se centran en el trabajo infantil, la inequidad de género y condiciones injustas de trabajo de miles de trabajadores, principalmente en el continente africano (Vásquez et al., 2019).

Peprah (2015), por ejemplo, analiza exhaustivamente como la calidad de vida de los productores de cacao ha sido tomada a la ligera, no se toma en cuenta la real importancia que tiene para el desarrollo el bienestar de los productores agrícolas. En las economías en desarrollo, la mayoría de la población vive de la agricultura, por lo que al hablar de agricultura sostenible y medios de vida sostenibles no se puede desconocer que ambos temas están estrechamente relacionados.

La producción de grano de cacao es una de las principales fuentes de ingresos para más de cinco millones de pequeños productores del mundo (Niether et al., 2019; Buyer, Baligar, He \& Arévalo-Gardini, 2017), para estos minifundistas los ingresos recibidos por concepto de la producción de cacao corresponden entre el $70 \%$ y $100 \%$ de los ingresos percibidos anualmente para su hogar (Adams et al., 2017); en total 50 millones de personas, aproximadamente, dependen del cacao y sus productos derivados (Quiroz \& Fogliano, 2018; Utomo et al., 2016); este sistema productivo está caracterizado por una fuerte inversión de mano de obra de tipo familiar (Vásquez et al., 2019).
El área total de las fincas típicas cacaoteras en Asia y África está entre dos y cuatro hectáreas, con una producción promedio de $300-400 \mathrm{~kg}$ de cacao seco fermentado en África y alrededor de $500 \mathrm{~kg}$ en Asia; en América los predios suelen ser más grandes con producciones de entre $500-600 \mathrm{~kg}$ ha-1 (Shavez et al., 2017).

Figura 2. Evolución de la producción de cacao en grano en el mundo, de 1961 a 2018.

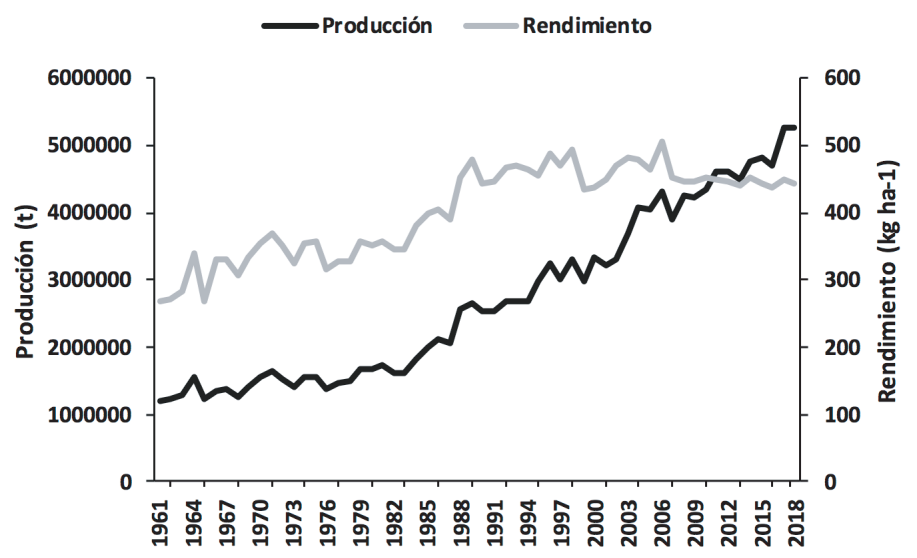

Fuente: elaboración propia con datos Faostat (2020).

Desde que la FAO lleva la estadística de la producción de cacao en grano (1961), hasta la última cosecha reportada (2018), se pasó de producir 1.186.364 t a 5.252.377 t (Figura 2), si bien parece un aumento considerable de más de cuatro millones de toneladas, estas no están representadas por un aumento en la productividad por unidad de área, que se mantiene por debajo de los 500 kg ha-1 año-1 (402,6 kg ha-1 promedio desde 1961-2018), sino en la necesidad de haber ampliado la frontera agrícola, pasando de 4.403 .484 ha en 1961 a 11.834 .970 ha en la cosecha del 2018.

Tothmihaly, Ingram y Von Cramon(2019) proponen tres alternativas que ayudan a mejorar los ingresos de los agricultores y la demanda mundial de producto: la primera está referida al aumento de las áreas cultivadas; la segunda menciona aumento en el uso de insumos intermedios (como fertilizantes, productos químicos y pesticidas), por último sugieren una eficiencia técnica, que se traduce en maximizar los productos usando un nivel fijo de insumos. Sin embargo, los mismos autores señalan que este tipo de opciones para incrementar la producción puede traer como consecuencia daños ambientales como pérdida de nutrientes, en la biodiversidad, aumento de polución y efectos directos en el cambio climático.

El manejo del cultivo como un agroecosistema es una alternativa que ha tomado fuerza. Kieck, Zug, Huamaní, Gómez y Cierjacks (2016) afirman que este tipo de cultivos juega un papel importante en la conservación de la vida del planeta. Existe una creciente evidencia de que esa biodiversidad 
podría ser benéfica para la producción agrícola, al crear agroecosistemas con una alta diversidad que pueden ser altamente productivos (ej. sistemas agroforestales).

Estos sistemas agroforestales (SAF) constituyen una alternativa ante el problema de los monocultivos, permiten que estos sean desplazados, dado que implican la combinación de árboles de tipo forestal con otros cultivos, lo que posibilita optimi

zar la producción mientras que se respeta el principio de obtener rendimientos sostenibles (Mata-Anchundiaet al., 2018).

\section{VI.Conclusiones.}

La anterior revisión permite identificar las dificultades a las que actualmente se enfrentan los productores mundiales de cacao. Temas limitantes como los metales pesados que se bioacumulan en el grano y pueden afectar la salud humana junto con plagas y enfermedades serán los desafíos por superar a corto plazo. Lo anterior es clave a la hora de tomar la decisión de establecer un cultivo de cacao.

Por otra parte, es clara la oportunidad que existe alrededor de este cultivo por la creciente demanda de productos procesados en países como Brasil, India y China. Estas economías emergentes han hecho que durante los últimos años el consumo se incremente y se presente un déficit en la producción requerida.

Producir cacao con enfoque agroforestal se convierte en una alternativa para los productores, para que su flujo de caja no dependa exclusivamente del cacao; además aporta a la conservación del ecosistema y reduce el uso de insumos externos.

\section{Referencias}

Acosta, N., De Vrieze, J., Sandoval, V., Sinche, D., Wierinck, I. \& Rabaey K. (2018). Cocoa Residues as Viable Biomass for Renewable Energy Production through Anaerobic Digestion. Bioresource Technology, 265, 568-572. Doi: https://doi.org/10.1016/j.biortech.2018.05.100.

Acosta, S. \& Pozo, P. (2013). Determinación de cadmio en la almendra de cacao (Theobroma cacao) de cinco fincas ubicadas en la vía Santo Domingo - Esmeraldas, mediante espectrofotometría de absorción atómica con horno de grafito. Revista Escuela de Ciencias Químicas Infoanalítica, 1(1), 69-82.

Adams, W., Acheampong, E., Kyereh, E. \& Kyereh, B. (2017). Farmers' Perspectives on Climate Change Manifestations in Smallholder Cocoa Farms and Shifts in Cropping Systems in the Forest-Savannah Transitional Zone of Ghana. Land Use Police, 66, 374-381. Doi: https://doi.org/10.1016/j.landusepol.2017.05.010.

Agronet, Ministerio de Agricultura y Desarrollo Rural. (2020).
Precio de referencia semanal de compra de cacao. Fuente industria -FEDECACAO- Exportadores. Recuperado de https://www.agronet.gov.co/Noticias/Paginas/Precio-dereferencia-semanal-de-compra-de-cacao---Fuente-Industria.aspx\#.

Agudelo-Castañeda, G. A., Cadena-Torres, J., Almanza-Mechán, P. J. \& Pinzón-Sandoval. E. H. (2018). Desempeño fisiológico de nueve genotipos de cacao (Theobroma cacao L.) bajo la sombra de tres especies forestales en Santander, Colombia. Revista Colombiana de Ciencias Hortícolas, 12(1), 223-232. Doi: https://doi.org/10.17584/rcch.2018v12i1.7341.

Akrofi, A., Amoako, I., Assuah, M. \& Asare, E. K. (2015). Black Pod Disease on Cacao (Theobroma cacao L.) in Ghana: Spread of Phytophthora megakarya and Role of Economic Plants in the Disease Epidemiology. Crop Protection, 72, 66-75. Doi: https://doi.org/10.1016/j.cropro.2015.01.015

Andres, C., Blaser, W. J., Dzahini-Obiatey, H. K., Ameyaw, G. A., Domfeh, O. K., Awiagah, M. A., Gattinger, A., Schneider, M., Offei, S. K. \& Six. J. (2018). Agroforestry Systems can mitigate the Severity of Cocoa Swollen Shoot Virus Disease. Agriculture, Ecosystems \& Environment, 252, 83-89. Doi: https://doi.org/10.1016/j.agee.2017.09.031.

Andres, C., Gattinger, A., Dzahini-Obiatey, H. K., Blaser, W. J. S., Offei, K. \& Six. J. (2017). Combatting Cocoa Swollen Shoot Virus Disease: What do we know? Crop Protection, 98, 76-84. Doi: https://doi.org/10.1016/j.cropro.2017.03.010.

Arévalo, E., Arévalo, C. O., Baligar, V. C. \& He. Z. L. (2017). Heavy Metal Accumulation in Leaves and Beans of Cacao (Theobroma cacao L.) in Major Cacao grown in Regions in Peru. Science of the Total Environment, 605-606, 792-800. Doi: https://doi.org/10.1016/j.scitotenv.2017.06.122.

Argüello, D., Chávez, E., Lauryssen, F., Vanderschueren, R., Smolders, E. \& Monyalvo, D. (2019). Soil Properties and Agronomic Factors Affecting Cadmium Concentrations in Cacao Beans: A Nationwide Survey in Ecuador. Science of the Total Environment, 649, 120-127. Doi: https://doi.org/10.1016/j.scitotenv.2018.08.292.

Arvelo, M. A., Delgado, T., Maroto, S., Rivera, J., Higuera, I. \& Navarro, A. (2016). Estado actual sobre la producción y el comercio del cacao en América. San José, Costa Rica: IICA.

Bailey, B. A., Ali, S., Strem, M. D. \& Meinhardt, L. W. (2018). Morphological Variants of Moniliophthora roreri on Artificial Media and the Biotroph/necrotrophy Shift. Fungal Biology, 122(7). 701-716. Doi: https://doi.org/10.1016/j.funbio.2018.03.003. 
Beg, M. S., Ahmad, S., Jan, K. \& Bashir, K. (2017). Status, Supply Chain and Processing of Cocoa. A Review. Trends in Food Science \& Technology, 66, 108-116. Doi: https://doi.org/10.1016/j.tifs.2017.06.007.

Blaser, W. J., Oppong, J., Yeboah, E. \& Six, J. (2017). Shade Trees have limited Benefits for Soil Fertility in Cocoa Agroforests. Agriculture, Ecosystems \& Environment, 243, 83-91. Doi: https://doi.org/10.1016/j.agee.2017.04.007.

Buyer, J. S., Baligar, V. C., He, Z. \& Arévalo-Gardini, E. (2017). Soil Microbial Communities under Cacao Agroforestry and Cover Crop Systems in Peru. Applied Soil Ecology, 120, 273-280. Doi: https://doi.org/10.1016/j.apsoil.2017.09.009.

Campos, R., Nieto, K. H. \& Oomah, B. D. (2018). Cocoa (Theobroma cacao L.) Podhusk: Renewable Source of Bioactive Compounds. Trends in Food Science \& Technology, 81, 172-184. Doi: https://doi.org/10.1016/j.tifs.2018.09.022.

Carabalí, A., Senejoa, C. E. \& Montes, M. (2018). Reconocimiento, daño y opciones de manejo de Carmenta foraseminis Eichlin (Lepidóptera: Sesiidae), perforador del fruto y semilla de cacao Theobroma cacao L. (Malvaceae). Mosquera, Colombia: Corporación Colombiana de Investigación Agropecuaria Agrosavia.

Cárdenas, N. J., Darghan, A., Sosa, M. D. \& Rodríguez, A. (2017). Análisis especial de la incidencia de enfermedades en diferentes genotipos de cacao (Theobroma cacao L.) en el yopal (Casanare), Colombia. Acta Biológica Colombiana, 22(2), 209-220. Doi: http://dx.doi.org/10.15446/abc.v22n2.61161.

Casteblanco, J.A. (2018). Técnicas de remediación de metales pesados con potencial aplicación en el cultivo de cacao. La Granja: Revista de Ciencias de la Vida, 27, 21-35. Doi: https://doi.org/10.17163/lgr.n27.2018.02.

Castro, E. M, Idrogo-Vázquez, G., Siche, R. \& Cárdenas, F. P. (2019). Formation of Aromatic Compounds Precursors during Fermentation of Criollo and Forastero Cocoa. Heliyon, 5(1), 1-29. Doi: https://doi.org/10.1016/j.heliyon.2019.e01157.

Chávez, E., He, Z. L., Stofella, P. J., Mylavarapu, R. S., Li, Y. C., Moyano, B. \& Baligar, V. C. (2015). Concentration of Cadmium in Cacao Beans and its Relationship with Soil Cadmium in Southern Ecuador. Science of Total Environment, 53, 205-214. Doi: https://doi.org/10.1016/j.scitotenv.2015.06.106.

Correa, J., Castro, S. \& Coy, J. (2014). Estado de la moniliasis del cacao causada por Moniliophthora roreri en Colombia. Revista Acta Agronómica, 63(4), 388-399.

Cubillos, G. (2017). Frosty Pod Rot, Disease that affects the
Cocoa (Theobroma cacao) Crops in Colombia. Crop Protection, 96, 77-82. Doi: https://doi.org/10.1016/j.cropro.2017.01.011.

Curry, G. N., Koczberski, G., Lummani, J., Nailina, R., Peter, E., McNally, G. \& Kuaimba, O. (2015). A Bridge too far? The Influence of Socio-cultural Values on the Adaptation Responses of Smallholders to a Devastating Pest outbreak in Cocoa. Global Environmental Change, 35, 1-11. Doi: https://doi.org/10.1016/j.gloenvcha.2015.07.012.

De Araújo, R. P., Furtado, A. A., Pinto, J., de Oliveira, R. A., Pinto, F., Ahnert, D. \& Baligar, V. (2017). Molecular and Morphophysiological Responses Cocoa Leaves with Different Concentrations of Anthocyan into Variations in Light Levels. Scientia Horticulturae, 224, 188-197. Doi: https://doi.org/10.1016/j.scienta.2017.06.008.

Díaz-Valderrama, J. R., \& Aime, M. C. (2016). The Cacao Pathogen Moniliophthora roreri (Marasmiaceae) produces Rhexolytic Thallic Conidia and their Size is influenced by Nuclear Condition. Mycoscience, 57(3), 208-216. Doi: https://doi.org/10.1016/j.myc.2016.01.004.

Ebagnerin, J., N'guessan, F., Martinez, A., Sey, B., Wowo, A. \& Gnessougou, N. (2015). Ecological Changes induced by Full-sun Cocoa Farming in Côte d'Ivoire. Global Ecology and Conservation, 3, 575-595. Doi: https://doi.org/10.1016/j.gecc0.2015.02.007.

Espinosa-Alzate, J.A. \& Ríos-Osorio, L.A. (2016). Caracterización de sistemas agroecológicos para el establecimiento de cacao (Theobroma cacao L.), en comunidades afrodescendientes del Pacífico colombiano (Tumaco-Nariño, Colombia). Acta Agronómica, 65, 211-217. Doi: http://dx.doi.org/10.15446/acag.v65n3.50714.

Evans, H. C. (2016). Frosty Pod Rot (Moniliophthoraroreri). Cacao Diseases, a History of Old Enemies and New Encounters. In B. A. Bailey \& L. W. Meinhardt (Eds.), (pp. 63-96). USA: Springer.

Faostat (2018). Estadísticas de la Organización de Naciones Unidas para la Alimentación FAO. Recuperado de http://www.fao.org/faostat

Faostat (2020). Estadísticas de la Organización de Naciones Unidas para la Alimentación FAO. Recuperado de http://www.fao.org/faostat

Fedecacao. (2019). Estadísticas y recaudo.

h t t p : / w ww.fedecaca o.com.co/portal/index.php/es/2015-02-12-17-20-59/nacionales 
Figueroa, W., Ramírez, J. A., Katil, A. \& Rieche, S. (2013). Efecto de las cepas nativas Paecilomyces sp. (Bainier) y Lecanicillium sp. (Zimm) en el control de Carmenta foraseminis Eichlin (Lepidoptera: Sesiidae) en cultivos de cacao (Theobroma cacao L.). Acta Agronómica, 62(3), 279-286.

Gramlich, A., Tandy, S., Andres, C., Chincheros, J., Armengot, L., Schneider, M. \& Schulin, R. (2017). Cadmium Uptake by Cocoa Trees in Agroforestry and Monoculture Systems under Conventional and Organic Management. Science of the Total Environment, 580, 677-686. Doi: https://doi.org/10.1016/j.scitotenv.2016.12.014.

Gramlich, A., Tandy, S., Gauggel, C., López, M., Perla, D., González, V. \& Schulin, R. (2018). Soil Cadmium Uptake by Cocoa in Honduras. Science of the Total Environment, 612, 370-378. Doi: https://doi.org/10.1016/j.scitotenv.2017.08.145.

Hirons, M., Robinson, E., McDermott, C., Morel, A., Asare, R., Boyd, E., Gonfa, T., Gole, T. W., Malhi, Y., Mason, J. \& Norris, K. (2018). Understanding Poverty in Cash-crop Agro-forestry Systems: Evidence from Ghana and Ethiopia. Ecological Economics, 154, 31-41. Doi: https://doi.org/10.1016/j.ecolecon.2018.07.021.

Huamaní, H. A., Huauya, M. A., Mansilla, L. G., Florida, N. \& Neira, G. M. (2012). Presencia de metales pesados en cultivo de cacao (Theobroma cacao L.) orgánico. Acta Agronómica, 61(4), 339-344.

Hulme, M. F., Salliss, D., Konneh, M. S., Dauda, P., Witcutt, E. \& Sanderson, F. J. (2018). Improving Cocoa Harvest can mitigate for Crop Damage by Wildlife in a Forest-Agriculture Matrix. Agriculture, Ecosystems \& Environment, 265, 236-243. Doi: https://doi.org/10.1016/j.agee.2018.06.019.

Instituto Colombiano Agropecuario -ICA-. (2012). Manejo fitosanitario del cultivo de cacao (Theobroma cacao L.) medidas para la temporada invernal. Bogotá D.C.: Produmedios.

Instituto Colombiano Agropecuario -ICA-. (2017). Reconozca los barrenadores de la mazorca del cacao. Recuperado de www.ica.gov.co

Instituto Nacional de Investigaciones Agrícolas -INIA-. (2004). Nuevo insecto perforador del fruto del cacao de importancia económica en Venezuela. Recuperado de www.inia.gob.ve

Instituto Nacional de vigilancia de medicamentos y alimentos. 12016. Programa nacional de vigilancia y control de cadmio en productos derivados del cacao (licor de cacao, chocolate de mesa, cocoa en polvo y chocolatina de leche). En. https://www.invima.gov.co/documents/20143/441038/Documento-tecnico-Cadmio-en-cacao.pdf/36200805-c21d-c444-ee08-4 7a0f6c8c230, Consulta. febrero de 2020.

Jaimes, Y. \& Aranzazu, F. (2010). Manejo de las enfermedades del cacao (Theobroma cacao L) en Colombia, con énfasis en monilial (Moniliophthora roreri). Bogotá D.C.: Corpoica.

Jaimes, Y., González, C., Rojas, J., Cornejo, O. E., Mideros, M. F., Restrepo, S., Cilas, C. \& Furtado, E. L. (2016). Geographic Differentiation and Population Genetic Structure of Moniliophthora roreri in the Principal Cocoa Production Areas in Colombia. Plant Disease Journal, 100(8), 1548-1558. Doi: https://doi.org/10.1094/PDIS-12-15-1498-RE.

Kieck, J. S., Zug, K. L., Huamaní, H. A., Gómez, R. \& Cierjacks, A. (2016). Plant Diversity Effects on Crop Yield, Pathogen Incidence, and Secondary Metabolism on Cacao Farms in Peruvian Amazonia. Agriculture, Ecosystems \& Environment, 222, 223-234. Doi: https://doi.org/10.1016/j.agee.2016.02.006.

Kongor, J., Hinneh, M., Van de Walle, D., Afoakwa, E. O., Boeckx, P. \& Dewettinck, K. (2016). Factors Influencing Quality Variation in Cocoa (Theobroma cacao) Bean Flavour Profile. A Review. Food Research International, 82, 44-52. Doi: https://doi.org/10.1016/j.foodres.2016.01.012.

Kruszewski, B., Obiedzinski, M. \& Kowalska, J. (2018). Nickel, Cadmium and Lead Levels in Raw Cocoa and Processed Chocolate Mass Materials from Three Different Manufacturers. Journal of Food Composition and Analysis, 66, 127-135. Doi: https://doi.org/10.1016/j.jfca.2017.12.012.

Lachenaud, Ph., Paulin, D., Ducamp, M. \& Thevenin, J. M. (2007). Twenty Years of Agronomic Evaluation of Wild Cocoa Trees (Theobroma cacao L.) from French Guiana. Scientia Horticulturae, 113(4), 313-321. Doi: https://doi.org/10.1016/j.scienta.2007.05.016.

Legavre, T., Ducamp, M., Sabau, X., Argout, X., Fouet, O., Dedieu, F., Surujdeo, S., Garcia, D., Paulin, D. \& Lanaud, C. (2015). Identification of Theobroma cacao Genes Differentially Expressed during Phytophthora megakarya Infection. Physiological and Molecular Plant Pathology, 92, 1-13. Doi: https://doi.org/10.1016/j.pmpp.2015.08.005.

Lewis, C., Lennon, A. M., Eudoxie, G. \& Umaharan, P. (2018). Genetic Variation in Bioaccumulation and Partioning of Cadmium in Theobroma cacao L. Science of the Total Environment, (640-641), 696-703. Doi: https://doi.org/10.1016/j.scitotenv.2018.05.365.

Lo Dico, G., Galvano, F., Dugo, G., D’ascenzi, C., Macaluso, A., Vella, A., Giangrosso, G., Cammilleri, G. \& Ferrantelli, V. (2018). Toxic Metal Levels in Cocoa Powder and Chocolate by ICP-MS 
Method after Microwave-Assisted Digestion. Food Chemistry, 245, 1163-1168. Doi: https://doi.org/10.1016/j.foodchem.2017.11.052.

Lu, F., Rodríguez, J., Van Damme, I., Westwood, N. J., Shaw, L., Robinson, J. S., Warren, G., Chatzifragkou, A. McQueen, S., Gómez, L., Faas, L., Balcombe. K., Srinivasan, C., Picchioni, F., Hadley, P. \& Charalampopoulos, D. (2018). Valorization Strategies for Cocoa Podhusk and its Fractions. Current Opinion in Green and Sustainable Chemistry, 14, 80-88. Doi: https://doi.org/10.1016/j.cogsc.2018.07.007.

Martínez, E. \& Pérez, L. (2015). Incidencia de enfermedades fúngicas en plantaciones de cacao de las provincias orientales de Cuba. Revista Protección Vegetal, 30(2), 87-96.

Mata-Anchundia, D., Rivero-Herrada, M., \& Segovia-Montalvan, E. 2018. Sistemas agroforestales con cultivo de cacao fino de aroma: entorno socio-económico y productivo. Revista cubana de ciencias forestales. 6 (1). 103-115.

McMahon, P., bin Purung, H., Lambert, S., Mulia, S., Nurlaila, Susilo, A.W., Sulistyowati, E., Sukamto, S., Israel, M., Saftar, A., Amir, A., Purwantara, A., Iswanto, A., Guest, D. \& Keane, P. (2015). Testing Local Cocoa Selections in Three Provinces in Sulawesi: (i) Productivity and Resistance to Cocoa Pod Borer and Phytophthora pod rot (black pod). Crop Protection, 70(28), 39. Doi: https://doi.org/10.1016/j.cropro.2015.01.001.

Mejía, L. A. \& Palencia, G. E. (2004). Una aproximación a los sistemas agroforestales con cacao. Bucaramanga, Colombia: Corpoica.

Montoya, I. A., Montoya, L. A. \& Lowy, P. D. (2015). Oportunidades para la actividad cacaotera en el municipio de Tumaco, Nariño, Colombia. Entramado, 11(1), 48-59. http://dx.doi.org/10.18041/entramado.2015v11n1.21107.

Ndoungue, M., Perchayo, S., Techou, Z., Nana, W. G., Nembot, C., Fontem, D. \& Ten Hoopen, G. M. (2018). The Impact of Soil Treatments on Black Pod Rot (caused by Phytophthora megakarya) of Cacao in Cameroon. Biological Control, 123, 9-17. Doi: https://doi.org/10.1016/j.biocontrol.2018.04.016.

Niether, W., Schneidewind, U., Armengot, L., Adamtey, N., Schneider, M. \& Gerold, G. (2017). Spatial-temporal Soil Moisture Dynamics under Different Cocoa Production Systems. Catena, 158, 340-349. Doi: https://doi.org/10.1015/j.catena.2017.07.011.

Niether, W., Schneidewind, U., Fuch, M., Schneider, M. \& Armengot, L. (2019). Below and Above Ground Production in Cocoa
Monocultures and Agroforestry Systems. Science of the Total Environment, 657, 558-567. Doi: https://doi.org/10.1016/j.scitotenv.2018.12.050.

Obok, E., Wetten, A. \& Allainguillaume, J. (2018). Electropenetrography Application and Molecular-based Virus Detection in Mealybug (Hemiptera: Pscudococcidae) Vectors of Cacao swollen shoot virus on Theobroma cacao L. Annals of Agricultural Sciences, 63(1), 55-65. Doi: https://doi.org/10.1016/j.aoas.2018.04.004.

Peprah, K. (2015). Sustainability of Cocoa Farmers' Livelihoods: A Case Study of Asunafo District, Ghana. Sustainable Production and Consumption, 4, 2-15. Doi: https://doi.org/10.1016/j.spc.2015.09.001.

Purwantara, A., McMahon, P., Susilo, A. W., Sukamto, S., Mulia, S., Nurlaila, Saftar, A., bin Purung, H., Lambert, S., Keane, P. \& Guest, D. (2015). Testing Local Cocoa Selections in Sulawesi: (ii) Resistance to Stem Canker and Pod Rot (Black Pod) Caused by Phytophthora palmivora. Crop Protection, 77(18), 26. Doi: https://doi.org/10.1016/j.cropro.2015.07.005.

Quiroz, C. N., \& Fogliano, V. (2018). Design Cocoa Processing towards Healthy Cocoa Products: The Role of phenolics and melanoidins. Journal of Functional Foods, 45, 480-490. Doi: https://doi.org/10.1016/j.jff.2018.04.031.

Ramírez, J. G. (2016). Pérdidas económicas asociadas a la pudrición de la mazorca del cacao causada por Phytophthorra spp., y Moniliophthora roreri (Cif y Par) Evans et al., en la hacienda Theobroma, Colombia. Revista de Protección Vegetal, 31(1), 42-49.

Rodríguez, H. S., Darghan, A. E. \& Henao, M. C. (2019). Spatial Regression Modeling of Soils with High Cadmium Content in a Cocoa Producing Area of Central Colombia. Geoderma Regional, 16. Doi: https://doi.org/10.1016/j.geodrs.2019.e00214.

Romero-Estévez, D., Yánez-Jácome, G. S., Simbaña-Farinango, K. \& Navarrete, H. (2019). Content and the Relationship between Cadmium, Nickel, and Lead Concentrations in Ecuadorian Cocoa Beans from Nine Provinces. Food Control, 106, 8. Doi. https://doi.org/10.1016/j.foodcont.2019.106750. Shavez, M., Ahmad, S., Jan, K. \& Bashir, K. (2017). Status, Supply Chain and Processing of Cocoa. A Review. Trends in Food Science \& Technology, 66, 108-116. Doi: https://doi.org/10.1016/j.tifs.2017.06.007. 
Singh, K., Sanderson, T., Field, D., Fidelis, C. \& Yinil, D. (2019). Soil Security for Developing and Sustaining Cocoa Production in Papua New Guinea. Geoderma Regional, 17. Doi: https://doi.org/10.1016/j.geodrs.2019.e00212.

Tothmihaly, A., Ingram, V. \& vonCramon, S. (2019). How can the Environmental Efficiency of Indonesian Cocoa Farms be increased? Ecological Economics, 158, 134-145. Doi: https://doi.org/10.1016/j.ecolecon.2019.01.004.

Utomo, B., Prawoto, A. A., Bonnet, S., Bangviwat, A. \& Gheewala, S. H. (2016). Environmental Performance of Cocoa Production from Monoculture and Agroforestry Systems in Indonesia. Journal of Cleaner Production, 134, 583-591. Doi: https://doi.org/10.1016/j.jclepro.2015.08.102.

Vásquez, Z. S., De Carvalho, D. P., Pereira, G. V. M., Vandenberghe, L. P. S., De Oliveira, P. Z., Tiburcio, P. B., Rogez, H. L. G., Goes, A. \& Soccol, C. R. (2019). Biotechnological Approaches for Cocoa Waste Management: A Review. Waste Management, 90, 72-83. Doi: https://doi.org/10.1016/j.wasman.2019.04.030.

Wessel, M. \& Quist, P.M. (2015). Cocoa Production in West Africa, A Review and Analysis of Recent Developments. NJAS - Wageningen Journal of Life Sciences, (74-75), 1-7. Doi: https://doi.org/10.1016/j.njas.2015.09.001.

Como Citar: Antolinez-Sandoval. E., Almanza-Merchán. P., Barona-Rodriguez. A., Polanco-Díaz. E., Serrano-Cely. P. (2020). Estado actual de la Cacaocultura: Una revisión de sus principales limitantes. Revista Ciencia y Agricultur a. 17(2) 1-11. D 0 l: h t t p s:// d o i . or g/10.19053/01228420.v17.n2.2020.10729 